\title{
Inflammatory Response in SARS-CoV-2 Infection of Patients with Schizophrenia and Long-Term Antipsychotic Treatment
}

\author{
Silvia Moga \\ Andreea Teodorescu \\ Petru Ifteni ip \\ Claudia Gavris \\ Paula-Simina Petric \\ Faculty of Medicine, Transilvania \\ University of Brasov, Brasov, Romania
}

Correspondence: Petru Ifteni Faculty of Medicine, Transilvania University of Brasov, Romania, 29th Eroilor Boulevard, Brasov, 500036, Romania

Tel +40 724993329

Email petru_ifteni@yahoo.com
Background: Schizophrenia patients are a population at particular risk of poor outcomes in COVID-19 infection. They have multiple comorbidities that have been identified as risk factors for severe COVID-19: diabetes, hypertension, chronic obstructive respiratory disease, and end-stage renal disease.

Aim: The aim of this research was to evaluate the inflammatory response and in-hospital mortality in schizophrenia patients compared to a control group without mental illness.

Methods: A total of 101 consecutive individuals with schizophrenia tested positive for COVID-19 was compared with 101 individuals without schizophrenia admitted in the same hospital. The number of severe cases and the number of deaths caused by SARS-CoV-2 were evaluated between April 2020 and April 2021.

Results: There were no deaths in the group of patients with schizophrenia. Although the group had a higher number of cases with pulmonary and metabolic comorbidities, in the group with SCZ there were fewer severe cases compared to the control group. The values of some markers of inflammation (CRP and fibrinogen) were significantly lower in SCZ patients. The duration from infection to diagnosis and the start of symptomatic treatment was shorter for the group with SCZ $(4.2 \pm 3.2$ vs $5.3 \pm 4.6, \mathrm{p}<0.05)$.

Conclusion: The main findings of the study were that vulnerable schizophrenia individuals on antipsychotic treatment showed a lower risk of SARS-CoV-2 severe infection and a likely better COVID-19 prognosis in a protective environment. Rapid access to specialists in case of need are factors that have determined the favorable evolution in a group considered high risk. It could be speculated that antipsychotics could play an important role in preventing SARS-CoV-2 severe manifestation and may exert protective effects against detrimental courses of COVID-19.

Keywords: schizophrenia, COVID-19, inflammation, antipsychotics

\section{Introduction}

Schizophrenia (SCZ) patients are a population at particular risk of poor outcomes in COVID-19 infection due to cognitive impairment, lack of insight and low economic status. They have multiple comorbidities that have been identified as risk factors for severe COVID-19: diabetes, hypertension, chronic obstructive respiratory disease, and end-stage renal disease. ${ }^{1}$ Previous studies have also shown reduced access to critical care for SCZ patients., ${ }^{2,3}$ Patients with schizophrenia that are long-term hospitalized may be at increased risk of adverse outcomes in the event of infection with SARS-CoV-2. 
Since the beginning of the pandemic, references have been made to the potential for morbidity and mortality induced by SARS-CoV-2 in schizophrenia ${ }^{4}$ as well as on the risks related to social distancing and the other restrictions imposed on the treatment. ${ }^{5}$ References have also been made to possible interactions between antipsychotics and treatment against SARS-CoV-2. ${ }^{6}$ Some authors have even questioned the antiviral potential of chlorpromazine. ${ }^{7}$

\section{Aims}

We aimed to establish whether health outcomes and care are different between patients with SCZ and patients without a diagnosis of severe mental illness. The primary objective was to compare inflammatory response and inhospital mortality between SCZ patients and patients without a diagnosis of severe mental illness.

\section{Methods}

This is a single center, prospective, cross-sectional study that includes 101 consecutive schizophrenia patients treated with oral antipsychotics in a long-term facility of Clinical Hospital of Psychiatry and Neurology of Brasov, Romania. This is an academic setting with 150 beds for acute patients and 300 beds for chronic patients. The study group of 101 schizophrenia patients was compared to a control group of 101 consecutive patients without psychiatric disorders such as: schizoaffective disorder, delusional disorder, bipolar disorder, dementia, intellectual disability. Patients with other psychiatric disorders (eg, anxiety disorder, personality disorder) that were treated off-label with antipsychotics were also excluded. All the patients in the study tested positive for COVID-19. All cases were admitted in a special designated unit of the same hospital as consequence of Romanian Government Policy at the beginning of Covid-19 pandemic in March 2020. The medical unit was declared "Hospital for infected patients with SARS-CoV-2" on April 7th 2020. All procedures were in accordance with the Declaration of Helsinki. Ethical approval was obtained from the local Committee for Ethical Research (protocol no. 23/06/2021). All subjects included in the study (both schizophrenia and control) provided written informed consent.

All data was collected from paper files and electronic files by trained psychiatrists with previous experience in this type of research. Demographic data, laboratory analyses including inflammatory markers, duration of hospitalization, severity of pulmonary forms of COVID-19 based on symptoms and imagistic (X-ray or CT scan for all cases), type of treatment administered, number of deaths were collected. Antipsychotic treatment (type, dose, and duration of administration) was recorded. According to the hospital's protocols, all blood samples for laboratory analyses were collected in the morning on empty stomach. All samples were venous blood collected in standardized kits.

SARS-CoV-2 infection was confirmed using 2 consecutive Polymerase Chain Reaction (PCR) tests (day 1 and day 5) performed by a specialist in laboratory medicine. Treatment was provided by a board certified specialist in infectious diseases, one cardiologist, and one specialist in internal medicine.

\section{Statistics}

We first compared baseline characteristics of both groups. Analysis of variance (ANOVA) and paired t-tests were used to compare means. Confidence intervals of proportions were calculated using Wilson's method. Data were analyzed using SPSS version 26 for Windows. All $P$-values were 2 -sided with a $P$ value $<0.05$ indicating statistical significance.

\section{Results}

One hundred and one patients with schizophrenia were admitted from a long-term hospitalization unit belonging to the same hospital between April 152020 and April 15 2021. Characteristics of the patients and controls are presented in Table 1.

All patients underwent comprehensive tests during hospitalization. The values obtained on admission (day 1) were considered the reference. We noticed the higher values of some inflammation markers in the control group compared to $\mathrm{SCZ}$ (CRP 39.11 \pm 73.04 vs 21.27 $\pm 51.28, \quad \mathrm{p}=0.04$; fibrinogen $485.06 \pm 176.45$ vs 372.71 $\pm 121.46, \mathrm{p}=0.0001)$. D-dimer levels were not statistically different. Physical health comorbidities were more frequent in SCZ group. Of the total sample, 5.94\% $(n=6)$ were obese, defined as a BMI (body mass index) $\geq 30$, $27.72 \%(\mathrm{n}=28)$ were overweight $(\mathrm{BMI}=25-29)$ and $66.34 \%(\mathrm{n}=67)$ had a normal BMI. There were 55 $(54.45 \%)$ cases with BMI $\geq 25$ in the control group. All the results are presented in Table 2.

The 2 analyzed groups (SCZ vs Control) did not differ in mean age nor in age groups (Figure 1)

In the SCZ group there are some modified values (eg, anemia, hyponatremia) but these elements are frequently 
Table I Patient's Characteristics

\begin{tabular}{|c|c|c|c|c|}
\hline \multicolumn{2}{|l|}{ Characteristics } & \multirow{3}{*}{$\begin{array}{l}\text { SCZ } \\
\mathbf{N}=101 \\
54.30(10.83)\end{array}$} & \multirow{3}{*}{$\begin{array}{l}\text { Control } \\
\mathbf{N}=101 \\
54.31(10.13)\end{array}$} & \multirow{3}{*}{$\begin{array}{l}p \text { value } \\
0.17\end{array}$} \\
\hline & & & & \\
\hline Age & Mean (SD) & & & \\
\hline Male & & 51 (50.49\%) & $53(52.47 \%)$ & 0.67 \\
\hline Length of stay & Mean (SD) & I5.II (7.47) & $15.50(7.96)$ & 0.71 \\
\hline \multirow[t]{3}{*}{ Severity of Covid-19 infection } & Mild & $86 ; 85.14 \%$ & $73 ; 72.27 \%$ & 0.02 \\
\hline & Moderate & $12 ; 11.87 \%$ & $22 ; 21.78 \%$ & 0.05 \\
\hline & Severe & $2 ; 1.98 \%$ & $6 ; 5.94 \%$ & 0.15 \\
\hline \multirow[t]{6}{*}{ Comorbidities } & Respiratory & $12 ; 11.88 \%$ & $4 ; 3.96 \%$ & 0.03 \\
\hline & Cardiovascular & $30 ; 29.70 \%$ & $42 ; 41.58 \%$ & 0.07 \\
\hline & Metabolic & $38 ; 37.62 \%$ & $25 ; 24.75 \%$ & 0.04 \\
\hline & Neurological & $3 ; 2.97 \%$ & $4 ; 3.96 \%$ & 0.70 \\
\hline & Others & II; $10.89 \%$ & $13 ; 12.87 \%$ & 0.66 \\
\hline & Without & $30 ; 29.70 \%$ & $38 ; 37.62 \%$ & 0.23 \\
\hline Deaths & & $0(0 \%)$ & $4(3.96 \%)$ & 0.04 \\
\hline
\end{tabular}

found in institutionalized patients. There were no statistically significant differences for BMI.

All SCZ patients received antipsychotic treatment. Despite the conflicting information at the beginning and during the COVID-19 pandemic, a significant number of patients $(n=21 ; 21.21 \%)$ continued treatment with clozapine while hospitalized. The reason was the history of aggression, violence or TRS (treatment-resistant schizophrenia). As patients under surveillance, stabilized on treatment, it was normal for them not to have high or maximum doses of antipsychotics. A large number of patients are still treated with haloperidol in our long-term unit $(\mathrm{n}=24,23.76 \%)$.

The distribution and types of antipsychotics and chlorpromazine dose equivalent are presented in Table 3.

According to the protocols for SARS-CoV-2 patients were treated with hydroxychloroquine, lopinavir/ritonavir, azithromycin, enoxaparin, etc. The anti SARS-CoV-2 treatment was prescribed and monitored by the infectious disease physician. Treatment also included dexamethasone, mucolytics, antitussives as shown in Table 4.

\section{Discussion}

To our knowledge, this is the first study to show a favorable evolution of schizophrenia patients infected with SARS-CoV-2, uninterruptedly treated with antipsychotics. The results could be considered surprising given the predicted risk of severe complications for this category at the onset of the COVID-19 pandemic in the spring of $2020 .{ }^{8}$ One of the possible reasons for the favorable evolution could be the fact that schizophrenia patients in the study group were repeatedly tested and therefore promptly diagnosed and quickly treated (after being tested positive for SARS-CoV-2) in their own hospital by a multidisciplinary team including an infectious disease specialist, an internal medicine specialist, neurologists and psychiatrists. This is in opposition to the higher hospitalization and mortality rates in patients with schizophrenia and SARS-CoV-2 infection that were reported in another study. ${ }^{9}$ Results of one study conducted in South Korea found that being tested positive for COVID-19 increased the risk for severity and mortality among people with mental disorders. ${ }^{10}$ Due to social isolation these individuals can present themselves for medical care only when their condition has already worsened. ${ }^{11,12}$

Inflammatory markers such as CRP, fibrinogen, and D-Dimers were collected from all patients. CRP and fibrinogen in the control group were higher compared to $\mathrm{SCZ}$ group; D-dimer levels were not statistically different. Evidence suggests that some individuals respond to SARS-CoV-2 infection through a "cytokine storm" type reaction, with characteristics similar to those encountered in septic shock of bacterial etiology. Associated biological markers may include increases in C-reactive protein and 
Table 2 Laboratory Results

\begin{tabular}{|c|c|c|c|c|c|}
\hline \multicolumn{3}{|l|}{ Parameters } & \multirow{3}{*}{$\begin{array}{l}\text { SCZ } \\
\mathbf{N}=101 \\
21.27(51.28)\end{array}$} & \multirow{3}{*}{$\begin{array}{l}\text { Control } \\
\mathbf{N}=101 \\
39.11 \text { (73.04) }\end{array}$} & \multirow{3}{*}{$\begin{array}{l}p \text { value } \\
0.04\end{array}$} \\
\hline \multicolumn{2}{|c|}{ Laboratory Analysis } & \multirow{2}{*}{$\begin{array}{l}\text { Normal Values } \\
0-5 \mathrm{MG} / \mathrm{L}\end{array}$} & & & \\
\hline CRP & Mean (SD) & & & & \\
\hline D-DIMER & Mean (SD) & 0-500 MG/ML & $858.14(1253.35)$ & $658.38(717.27)$ & 0.16 \\
\hline ESR & Mean (SD) & $2-20 \mathrm{MM} / \mathrm{H}$ & $25.39(20.80)$ & $31.27(24.66)$ & 0.06 \\
\hline WBC & Mean (SD) & $4-10 \times 10^{9} / \mathrm{L}$ & $6.58(2.62)$ & $7.42(3.11)$ & 0.03 \\
\hline FIBRINOGEN & Mean (SD) & $200-400 \mathrm{MG} / \mathrm{DL}$ & $372.71(121.46)$ & $485.06(176.45)$ & 0.0001 \\
\hline BAS & Mean (SD) & $0.0-0.10 \times 10^{9} / \mathrm{L}$ & $0.02(0.01)$ & $0.04(0.19)$ & 0.29 \\
\hline NEU & Mean (SD) & $2.0-7.0 \times 10^{9} / \mathrm{L}$ & $4.38(3.85)$ & $4.89(2.94)$ & 0.29 \\
\hline EOS & Mean (SD) & $0.02-0.5 \times 10^{9} / \mathrm{L}$ & $0.09(0.11)$ & $0.05(0.06)$ & 0.001 \\
\hline LYM & Mean (SD) & $0.8-4.0 \times 10^{9} / \mathrm{L}$ & $1.95(0.07)$ & $\mathrm{I} .88(0.74)$ & 0.34 \\
\hline MON & Mean (SD) & $0.12-1.2 \times 10^{9} / \mathrm{L}$ & $0.51(0.25)$ & $0.54(0.21)$ & 0.33 \\
\hline RBC & Mean (SD) & $4.39-5.5 \times 10^{12} / \mathrm{L}$ & $4.33(0.5 \mathrm{I})$ & $4.53(0.61)$ & 0.01 \\
\hline HGB & Mean (SD) & $12-16 \mathrm{G} / \mathrm{DL}$ & $13.45(1.47)$ & $13.99(1.36)$ & 0.007 \\
\hline $\mathrm{MCV}$ & Mean (SD) & $80-100 \mathrm{FL}$ & $90.38(5.37)$ & $89.49(6.19)$ & 0.27 \\
\hline $\mathrm{MCH}$ & Mean (SD) & $27-34$ PG & $31.25(2.17)$ & $30.70(2.36)$ & 0.08 \\
\hline $\mathrm{MCHC}$ & Mean (SD) & $32-36 \mathrm{G} / \mathrm{DL}$ & $34.52(0.73)$ & $34.29(0.89)$ & 0.04 \\
\hline RDW-CV & Mean (SD) & $11-16 \%$ & $13.97(1.59)$ & $13.55(0.94)$ & 0.02 \\
\hline RDW-SD & Mean (SD) & $35-56 \mathrm{FL}$ & $44.97(4.65)$ & $43.10(3.36)$ & 0.001 \\
\hline HCT & Mean (SD) & $36-48 \%$ & $38.93(4.2)$ & $40.72(3.96)$ & 0.002 \\
\hline PLT & Mean (SD) & $150-400 \times 10^{9} / \mathrm{L}$ & $220.13(69.87)$ & 247.44 (99.57) & 0.02 \\
\hline MPV & Mean (SD) & $6.5-12 \mathrm{FL}$ & $9.99(1.21)$ & $9.98(0.98)$ & 0.94 \\
\hline PCT & Mean (SD) & $0.108-0.282 \%$ & $0.21(0.06)$ & $0.24(0.08)$ & 0.002 \\
\hline TGP & Mean (SD) & $0-31 \mathrm{U} / \mathrm{L}$ & $23.43(22.13)$ & $34.11(25.10)$ & 0.001 \\
\hline TGO & Mean (SD) & $0-38 \mathrm{U} / \mathrm{L}$ & $26.98(24.18)$ & $29.70(18.93)$ & 0.37 \\
\hline GLU & Mean (SD) & 74-106 MG/DL & I $20.83(39.4)$ & $126.98(43.47)$ & 0.29 \\
\hline CREA & Mean (SD) & $0.5-0.9 \mathrm{MG} / \mathrm{DL}$ & $0.89(0.43)$ & $0.98(0.87)$ & 0.35 \\
\hline UREA & Mean (SD) & I6.6-48.5 MG/DL & $31.42(18.24)$ & $32.86(18.03)$ & 0.57 \\
\hline GGT & Mean (SD) & $0-40 \mathrm{U} / \mathrm{L}$ & $45.81(51.48)$ & $73.60(110.50)$ & 0.02 \\
\hline PHOSPHATASE & Mean (SD) & $35-104 \mathrm{U} / \mathrm{L}$ & $71.68(18.58)$ & $83.26(18.19)$ & 0.0001 \\
\hline HDLC & Mean (SD) & 45-65 MG/DL & $41.27(9.26)$ & $42.33(15.87)$ & 0.56 \\
\hline LDL & Mean (SD) & $0-100 \mathrm{MG} / \mathrm{DL}$ & I I 6.39 (47.55) & 120.68 (54.69) & 0.5526 \\
\hline TRIG & Mean (SD) & $0-150 \mathrm{MG} / \mathrm{DL}$ & $160.84(82.06)$ & I 75.5 (89.73) & 0.22 \\
\hline AMYL & Mean (SD) & $28-100 \mathrm{U} / \mathrm{L}$ & $93.09(83.10)$ & $64.47(20.29)$ & 0.0009 \\
\hline $\mathrm{K}+$ & Mean (SD) & 3.5-5.1 MMOL/L & $4(0.5)$ & $4.05(0.47)$ & 0.46 \\
\hline $\mathrm{NA}+$ & Mean (SD) & |36-145 MMOL/L & 134.74 (4.12) & $|36.6|(2.99)$ & 0.0003 \\
\hline
\end{tabular}

Abbreviations: CRP, C-reactive protein; ESR, Erythrocyte sedimentation rate; WBC, White blood cell; BAS, Basophils; NEU, Neutrophils; EOS, Eosinophils; LYM, Lymphocytes; MON, Monocytes; RBC, Red blood cell; HGB, Hemoglobin; MCV, Mean corpuscular volume; MCH, Mean corpuscular hemoglobin; MCHC, Mean corpuscular hemoglobin concentration; RDW-CV, Red cell distribution width; RDW-SD, Red cell distribution width (standard deviation); HCT, Hematocrit; PLT, Thrombocytes; MPV, Mean platelet volume; PCT, Procalcitonin; TGP, Alanine aminotransferase; TGO, Aspartate aminotransferase; GLU, Glucose; CREA, Creatinine; GGT, Gamma-glutamyl transferase; HDLC, High-density lipoprotein; LDL, Low-density lipoprotein; TRIG, Triglycerides; AMYL, Amylase; K+, Potassium; NA+, Sodium.

ferritin, which appear to correlate with disease severity and mortality. ${ }^{13}$ In patients with COVID-19, the values of CRP at admission correlate with the severity of the disease and tend to be a good predictor of complications. ${ }^{14,15}$ In our study, the values of CRP were smaller in SCZ group compared with controls.

Ruan et al found that CRP levels correlate with the risk of mortality (surviving patients had a mean CRP of $4040 \mathrm{mg} / \mathrm{L}$, with an interquartile range of $1010-60 \mathrm{mg} / \mathrm{L}$, while non-survivors had an average of $125 \mathrm{mg} / \mathrm{L}$ with an interval interquartile range of $\sim 60-160 \mathrm{mg} / \mathrm{L}) .{ }^{16}$ Young et al found lower levels of CRP in patients who did not require additional oxygen. ${ }^{17}$ Our findings confirmed these results since only one patient with schizophrenia required oxygen therapy. Many patients with severe forms of COVID-19 infection have coagulation abnormalities that mimic other systemic coagulopathies associated with severe infections, such as disseminated intravascular 


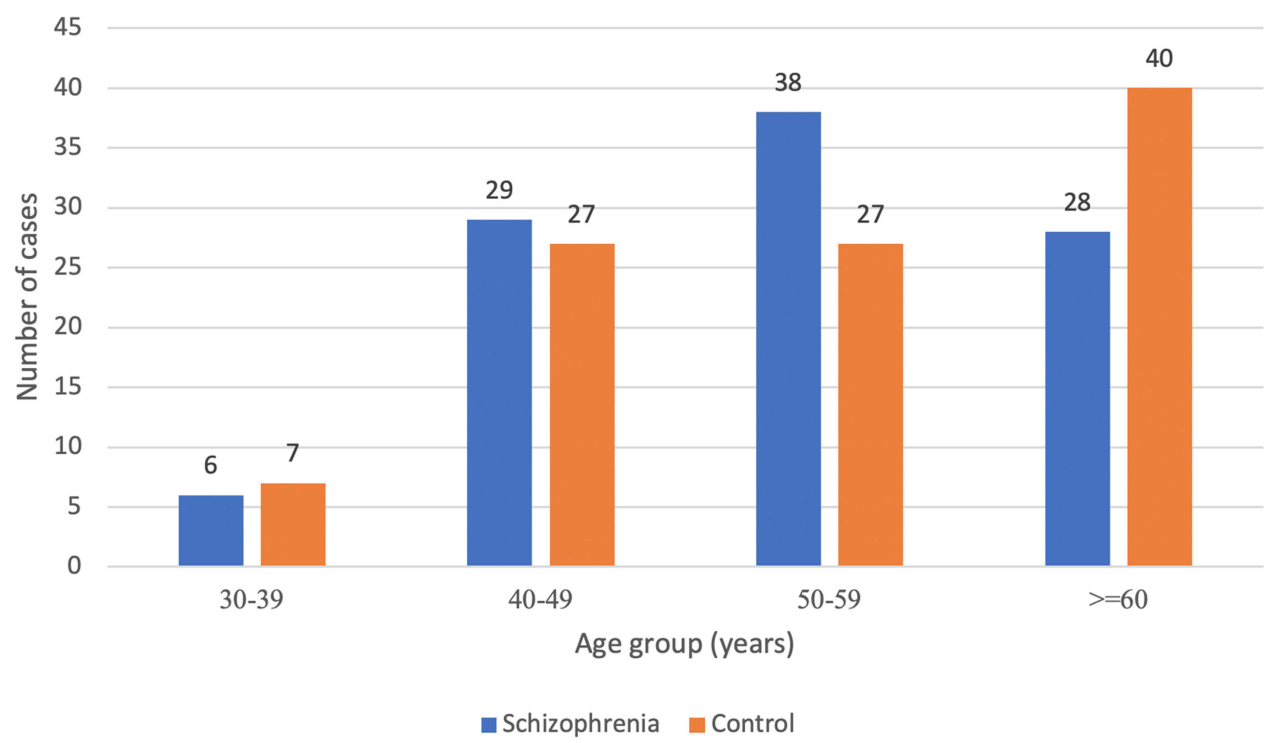

Figure I Age group.

coagulation or thrombotic microangiopathy. COVID-19 coagulopathy has distinct characteristics and is associated with an increased risk of death, causing venous and arterial thromboembolic complications. ${ }^{18}$ The most typical finding in patients with COVID-19 and coagulopathy is an increased concentration of D-dimers, a relatively modest decrease in platelet count, and a prolongation of prothrombin time. Studies have reported an increase in D-dimer and fibrinogen levels in the early stages of COVID-19, with a 3- to 4-fold increase in D-dimer levels being linked to poor prognosis. In addition, underlying diseases such as diabetes, cancer, stroke and pregnancy can trigger an increase in D-dimer levels in patients with COVID-19. ${ }^{19}$

Table 3 Antipsychotics Used in Schizophrenia Patients with COVID

\begin{tabular}{|l|l|l|}
\hline Antipsihotic Type & N, \% & CPZ Equivalent [mg] \\
\hline SGA & $25(25.25 \%)$ & $244(100.33)$ \\
\hline Olanzapine & $21(21.21 \%)$ & $245.65(102.43)$ \\
Clozapine & $12(12.12 \%)$ & $279.17(149.93)$ \\
Risperidone & $5(5.05 \%)$ & $746.67(292.12)$ \\
Quetiapine & $4(4.04 \%)$ & $200(80)$ \\
Amisulprid & $1(1.01 \%)$ & 199.99 \\
Aripiprazole & \multicolumn{3}{|l}{} \\
\hline FGA & $24(23.76 \%)$ & $259.26(119.32)$ \\
\hline Haloperidol & $3(3.03 \%)$ & $266.67(115.47)$ \\
Zuclopixol & $4(4.04 \%)$ & $244.56(104.22)$ \\
Flupenthixol &
\end{tabular}

Numerous studies have found that patients with schizophrenia have elevated blood levels of inflammatory cytokines and higher concentrations of these cytokines than controls during exacerbation of symptoms, but there was no difference found during the clinical stability periods. ${ }^{20}$ Our results confirmed that hypothesis.

Physical health comorbidities were more frequent in SCZ group. Pulmonary and metabolic comorbidities were significantly increased in the group of patients with schizophrenia, they being more prone to associate pulmonary, metabolic, cardiovascular comorbidities. ${ }^{21,22}$ They smoke excessively, ${ }^{23}$ have a sedentary lifestyle ${ }^{24}$ with an unbalanced diet ${ }^{25}$ which predisposes them to severe cardiac events and sudden death. ${ }^{26}$ The fact that patients were already hospitalized and their comorbidities were monitored, treated and stable, the fact that they live in a controlled environment with a caloric diet and also with smoking restrictions, can all be considered factors that have contributed to a low level of mortality and to full recovery after SARS-CoV-2 infection.

There were no statistically significant differences in BMI in the two groups, and the percentage of obese patients in the SCZ group was relatively small (5.94\%); therefore, this aggravating factor was not remarkable. Obesity is known to be an important risk factor for an unfavorable outcome in COVID patients, ${ }^{27,28}$ especially for those associating a severe mental illness. ${ }^{29}$

Last but not least, patients in the schizophrenia group received regular antipsychotic treatment in the last 3 months before infection. Despite the lack of data on this 
Table 4 Symptomatic Treatment

\begin{tabular}{|l|l|l|l|}
\hline Type of Treatment & SCZ $\mathbf{n = 1 0 I}$ & Control $\mathbf{n = 1 0 I}$ & P value \\
\hline Oxygen support & $1(0.99 \%)$ & $7(6.93 \%)$ & 0.0308 \\
Hydroxychloroquine & $0(0 \%)$ & $4(3.96 \%)$ & 0.0439 \\
Lopinavir/ritonavir & $2(1.98 \%)$ & $14(13.86 \%)$ & 0.0018 \\
Azithromycin & $29(28.71 \%)$ & $19(18.81 \%)$ & 0.0992 \\
Paracetamol & $79(78.21 \%)$ & $77(76.23 \%)$ & 0.7379 \\
Dexamethasone & $24(23.76 \%)$ & $17(16.83 \%)$ & 0.2219 \\
Anticoagulant & $39(38.61 \%)$ & $46(45.54 \%)$ & 0.3197 \\
Antibiotics & $26(25.74 \%)$ & $31(30.69 \%)$ & 0.4356 \\
Mucolitycs & $15(14.85 \%)$ & $1(0.99 \%)$ & 0.0003 \\
Antitussive & $15(14.85 \%)$ & $2(1.98 \%)$ & 0.0010 \\
Analgesics & $4(3.96 \%)$ & $8(7.92 \%)$ & 0.2350 \\
\hline
\end{tabular}

topic, we can assume that antipsychotics could have a protective effect against severe forms of SARS-CoV-2. Future studies will be able to bring new data in this field.

The study has several limitations that should be mentioned. One could be the relative small number of patients and the short duration of monitoring. Another limitation is that it was not a randomized study. The worldwide absolute mortality data suggest that COVID-19 infection may have different impacts across countries due to multiple factors (climate, facility organization, COVID-19 public management strategies, vaccination and the variant of SARS-CoV-2 virus). Since not all the factors and mechanisms of injuries, drug interactions, comorbidities and treatment involved in the complex network of COVID-19 infection have been taken into account, our results may be extrapolated partially in other countries. ${ }^{30}$

\section{Conclusion}

The main findings of the study were that vulnerable schizophrenia individuals on antipsychotic treatment showed a lower risk of SARS-CoV-2 severe infection and a likely better prognosis in a protective environment. It could be speculated that antipsychotics could play an important role preventing SARS-CoV-2 severe manifestation and may exert protective effects against detrimental courses of COVID-19. The results of the present study have to be taken judiciously, since not all the factors involved in the complex network of COVID-19 infection have been taken into account.

\section{Abbreviations}

SCZ, schizophrenia; ANOVA, analysis of variance; PCR, polymerase chain reaction; BMI, body mass index; TRS, treatment-resistant schizophrenia.

\section{Data Sharing Statement}

All relevant data are within the manuscript.

\section{Ethical Approval and Consent to Participate}

Ethical clearance was secured by the Ethical Committee of Clinical Hospital of Psychiatry and Neurology of Brasov, Romania. Informed written consent was taken. Confidentiality of the information was maintained and the data were recorded anonymously throughout the study. This study was conducted in accordance with the Declaration of Helsinki

\section{Acknowledgments}

The authors' gratitude goes to all the staff.

\section{Author Contributions}

All authors made substantial contributions to conception and design, acquisition of data, or analysis and interpretation of data; took part in drafting the article or revising it critically for important intellectual content; agreed to submit to the current journal; gave final approval of the version to be published; and agree to be accountable for all aspects of the work.

\section{Funding}

There is no funding to report.

\section{Disclosure}

The authors report no conflicts of interest in this work 


\section{References}

1. Kozloff N, Mulsant BH, Stergiopoulos V, Voineskos AN. The COVID-19 global pandemic: implications for people with schizophrenia and related disorders. Schizophr Bull. 2020;46(4):752-757. PMID: 32343342; PMCID: PMC7197583. doi:10.1093/schbul/sbaa051

2. Fond G, Salas S, Pauly V, et al. End-of-life care among patients with schizophrenia and cancer: a population-based cohort study from the French national hospital database. Lancet Public Health. 2019;4(11) e583-e591. PMID: 31677777. doi:10.1016/S2468-2667(19)30187-2

3. Melamed OC, Hahn MK, Agarwal SM, Taylor VH, Mulsant BH, Selby P. Physical health among people with serious mental illness in the face of COVID-19: concerns and mitigation strategies. Gen Hosp Psychiatry. 2020;66:30-33. PMID: 32645586; PMCID PMC7831754. doi:10.1016/j.genhosppsych.2020.06.013

4. Nemani K, Li C, Olfson M, et al. Association of psychiatric disorders with mortality among patients with COVID-19. JAMA Psychiatry. 2021;78(4):380-386. doi:10.1001/jamapsychiatry.2020.4442

5. Ifteni P, Dima L, Teodorescu A. Long-acting injectable antipsychotics treatment during COVID-19 pandemic - a new challenge. Schizophr Res. 2020;220:265-266. PMID: 32349886; PMCID PMC7185008. doi:10.1016/j.schres.2020.04.030

6. Plasencia-García BO, Rodríguez-Menéndez G, Rico-Rangel MI, RubioGarcía A, Torelló-Iserte J, Crespo-Facorro B. Drug-drug interactions between COVID-19 treatments and antipsychotics drugs: integrated evidence from 4 databases and a systematic review. Psychopharmacology. 2021;238(2):329-340. doi:10.1007/s00213-020-05716-4

7. Plaze M, Attali D, Petit AC, et al. Repurposing chlorpromazine to treat COVID-19: the reCoVery study. Encephale. 2020;46 (3):169-172. doi:10.1016/j.encep.2020.05.006

8. Maripuu M, Bendix M, Öhlund L, Widerström M, Werneke U. Death associated with coronavirus (COVID-19) infection in individuals with severe mental disorders in Sweden during the early months of the outbreak - an exploratory cross-sectional analysis of a population-based register study. Front Psychiatry. 2021;11:1538. doi:10.3389/fpsyt.2020.609579

9. Fond G, Pauly V, Leone M, et al. Disparities in intensive care unit admission and mortality among patients with schizophrenia and COVID-19: a national cohort study. Schizophr Bull. 2021;47 (3):624-634. PMID: 33089862; PMCID: PMC7665717. doi:10.1093/ schbul/sbaa 158

10. Lee SW, Yang JM, Moon SY, et al. Association between mental illness and COVID-19 susceptibility and clinical outcomes in South Korea: a nationwide cohort study. Lancet Psychiatry. 2020;7 (12):1025-1031. PMID: 32950066; PMCID: PMC7498216. doi:10.1016/S2215-0366(20)30421-1

11. Wang Q, Xu R, Volkow ND. Increased risk of COVID-19 infection and mortality in people with mental disorders: analysis from electronic health e United States. World Psychiatry. 2020;20(1):124-130. doi:10.1002/wps.20806

12. Fond G, Pauly V, Orleans V, et al. Increased in-hospital mortality from COVID-19 in patients with schizophrenia. Encephale. 2021;47 (2):89-95. PMID: 32933762; PMCID: PMC7392112. doi:10.1016/j. encep.2020.07.003

13. Fu L, Wang B, Yuan T, et al. Clinical characteristics of coronavirus disease 2019 (COVID-19) in China: a systematic review and meta-analysis. J Infect. 2020;80(6):656-665. PMID: 32283155; PMCID: PMC7151416. doi:10.1016/j.jinf.2020.03.041

14. Luo X, Zhou W, Yan X, et al. Prognostic value of C-reactive protein in patients with coronavirus 2019. Clin Infect Dis. 2020;71(16):2174-2179. PMID: 32445579; PMCID: PMC7314209. doi:10.1093/cid/ciaa641

15. Wang M, Zhu Q, Fu J, Liu L, Xiao M, Du Y. Differences of inflammatory and non-inflammatory indicators in Coronavirus disease-19 (COVID-19) with different severity. Infect Genet Evol. 2020;85:104511. PMID: 32858231; PMCID: PMC7448737. doi:10.1016/j.meegid.2020.104511
16. Ruan Q, Yang K, Wang W, Jiang L, Song J. Clinical predictors of mortality due to COVID-19 based on an analysis of data of 150 patients from Wuhan, China. Intensive Care Med. 2020;46 (5):846-848. Erratum in: Intensive Care Med. 2020 Apr 6. PMID: 32125452; PMCID: PMC7080116. doi:10.1007/s00134-020-05991-x

17. Young BE, Ong SWX, Kalimuddin S, et al.; Singapore 2019 Novel Coronavirus Outbreak Research Team. Epidemiologic features and clinical course of patients infected with SARS-CoV-2 in Singapore. JAMA. 2020;323(15):1488-1494. PMID: 32125362; PMCID: PMC7054855. doi:10.1001/jama.2020.3204

18. Levi M, Thachil J, Iba T, Levy JH. Coagulation abnormalities and thrombosis in patients with COVID-19. Lancet Haematol. 2020;7(6): e438-e440. doi:10.1016/S2352-3026(20)30145-9)

19. Rostami M, Mansouritorghabeh H. D-dimer level in COVID-19 infection: a systematic review. Expert Rev Hematol. 2020;13 (11):1265-1275. PMID: 32997543. doi:10.1080/17474086.20 20.1831383

20. Miller BJ, Goldsmith DR. Inflammatory biomarkers in schizophrenia: implications for heterogeneity and neurobiology. Biomark Neuropsychiatry. 2019;1:100006. doi:10.1016/j.bionps.2019.100006

21. Jaen-Moreno MJ, Feu N, Del Pozo GI, et al. Chronic obstructive pulmonary disease in severe mental illness: a timely diagnosis to advance the process of quitting smoking. Eur Psychiatry. 2021;64 (1):e22. PMID: 33632347; PMCID: PMC8057420. doi:10.1192/j. eurpsy.2021.12

22. Mitchell AJ, Vancampfort D, Sweers K, van Winkel R, Yu W, De Hert M. Prevalence of metabolic syndrome and metabolic abnormalities in schizophrenia and related disorders-a systematic review and meta-analysis. Schizophr Bull. 2013;39(2):306-318. PMID: 22207632; PMCID: PMC3576174. doi:10.1093/schbul/sbr148

23. Krieger I, Tzur Bitan D, Comaneshter D, Cohen A, Feingold D. Increased risk of smoking-related illnesses in schizophrenia patients: a nationwide cohort study. Schizophr Res PMID: 31395488. 2019;212:121-125. doi:10.1016/j.schres.2019.07.058

24. Vancampfort D, Firth J, Schuch FB, et al. Sedentary behavior and physical activity levels in people with schizophrenia, bipolar disorder and major depressive disorder: a global systematic review and meta-analysis. World Psychiatry. 2017;16(3):308-315. PMID: 28941119; PMCID: PMC5608847. doi:10.1002/wps.20458

25. Dipasquale S, Pariante CM, Dazzan P, Aguglia E, McGuire P, Mondelli V. The dietary pattern of patients with schizophrenia: a systematic review. J Psychiatr Res. 2013;47(2):197-207. PMID: 23153955. doi:10.1016/j.jpsychires.2012.10.005

26. Ifteni P, Correll CU, Burtea V, Kane JM, Manu P. Sudden unexpected death in schizophrenia: autopsy findings in psychiatric inpatients. Schizophr Res. 2014;155(1-3):72-76. PMID: 24704220. doi:10.1016/j.schres.2014.03.011

27. Kompaniyets L, Goodman AB, Belay B, et al.; Centers for Disease Control and Prevention. Body mass index and risk for COVID-19related hospitalization, intensive care unit admission, invasive mechanical ventilation, and death - United States, MarchDecember 2020. MMWR. 2021;70(10):355-361.

28. Lighter J, Phillips M, Hochman S, et al. Obesity in patients younger than 60 years is a risk factor for COVID-19 hospital admission. Clin Infect Dis. 2020;71(15):896-897. doi:10.1093/cid/ciaa415

29. Hert DE, Correll CU, Bobes J, et al. Physical illness in patients with severe mental disorders. I. Prevalence, impact of medications and disparities in health care. World Psychiatry. 2011;10(1):52-77.

30. Mishra AK, Lal A, Sahu KK, George AA, Sargent J. Letter to the Editor regarding "Neurological impact of Coronavirus disease (COVID-19): practical considerations for the neuroscience community". World Neurosurg. 2020;142:533-534. doi:10.1016/j. wneu.2020.05.089 


\section{Publish your work in this journal}

Neuropsychiatric Disease and Treatment is an international, peerreviewed journal of clinical therapeutics and pharmacology focusing on concise rapid reporting of clinical or pre-clinical studies on a range of neuropsychiatric and neurological disorders. This journal is indexed on PubMed Central, the 'PsycINFO' database and CAS, and is the official journal of The International Neuropsychiatric Association (INA). The manuscript management system is completely online and includes a very quick and fair peer-review system, which is all easy to use. Visit http://www.dovepress.com/testimonials.php to read real quotes from published authors. 